\title{
QUOTIENT RINGS OF RINGS GENERATED BY FAITHFUL CYCLIC MODULES
}

\author{
GARY F. BIRKENMEIER
}

\begin{abstract}
A ring $R$ is said to be generated by faithful cyclics (right finitely pseudo-Frobenius), denoted by right GFC (FPF), if every faithful cyclic (finitely generated) right $R$-module generates the category of right $R$-modules. A fundamental result in FPF ring theory, due to $\mathrm{S}$. Page, is that if $R$ is a right nonsingular right FPF ring, then $Q_{r}(R)$ is FPF. In this paper we generalize this result by providing a necessary and sufficient condition for a right nonsingular right GFC ring to have an FPF maximal right quotient ring.
\end{abstract}

Introduction. The class of right GFC rings includes right FPF rings, commutative rings (thus every ring has a GFC subring-its center), strongly regular rings, and regular right continuous rings of bounded index [3, Proposition 1.2 and Corollary 3.7]. To see that the class of right GFC rings is a nontrivial generalization of the class of right FPF rings, one need only recall that a commutative domain is FPF if and only if it is Prüfer, and a strongly regular ring is FPF if and only if it is selfinjective [8].

All rings are associative with unity. The following notation is used: $R$ is a ring, $Q_{r}(R)$ is the maximal right quotient ring of $R, \operatorname{Tr}_{R}(M)$ is the trace ideal of $M$ in $R$, $\underline{l}_{R}(X)\left[\underline{r}_{R}(X)\right]$ is the left [right] annihilator of $X$ in $R$. Two-sided concepts which are unmodified by the words "left" or "right" mean both sides are satisfied (e.g., $R$ is GFC means $R$ is right and left GFC). From [ 5 and 12] a ring $R$ is (quasi-) Baer if the right annihilator of every (ideal) nonempty subset of $R$ is a direct summand of $R$. We note that any right nonsingular right FPF ring is quasi-Baer $[7$, p. 168; $\mathbf{8}$, Theorem 3.3]. The basic references are $[\mathbf{1}, \mathbf{8}, \mathbf{1 0}$, and 17]. The following lemma appears in [3, Lemma 1.1] but is included here since it will be used repeatedly throughout the sequel.

LEMMA 1. $R$ is right GFC if and only if for every right ideal $X$ of $R$ such that $X$ contains no nonzero ideal then $\underline{l}(X) R=R$.

The following theorem generalizes [8, Corollary 5.11D and 3, Theorem 3.5].

THEOREM 2. Let $R$ be a right selfinjective ring. Then $R$ is right GFC if and only if $R$ is right FPF.

ProOF. Assume $R$ is right GFC. Let $M=\sum_{i=1}^{n} x_{i} R$ be a finitely generated faithful $R$-module, where $\left\{x_{i} \mid i=1,2, \ldots, n\right\}$ is a set of generators of $M$. Since $M$ is faithful, $\bigcap_{i=1}^{n} \underline{r}\left(x_{i}\right)$ contains no nonzero ideals. By Lemma 1, [1, p. 113, Exercise 8

Received by the editors March 21, 1986.

1980 Mathematics Subject Classification (1985 Revision). Primary 16A08; Secondary 16A36.

Key words and phrases. Maximal right quotient ring, FPF, GFC, selfinjective, nonsingular, faithful, generator. 
and 17, p. 275, Proposition 2.2],

$$
R=\underline{l}\left(\bigcap_{i=1}^{n} \underline{r}\left(x_{i}\right)\right) R=\sum_{i=1}^{n} \underline{l}\left(\underline{r}\left(x_{i}\right)\right) R=\sum_{i=1}^{n} \operatorname{Tr}_{R}\left(x_{i} R\right) .
$$

But $R$ is right selfinjective, so $\operatorname{Tr}_{R}\left(x_{i} R\right) \subseteq \operatorname{Tr}_{R}(M)$ for all $i=1,2, \ldots, n$. Thus $\operatorname{Tr}_{R}(M)=R$. From [1, Proposition 8.21], $R$ is right FPF. The converse is obvious.

THEOREM 3. Let $R$ be a right GFC ring such that whenever $I$ is a nonzero ideal of $R$, then $I Q_{r}(R)$ (i.e., the right ideal of $Q_{r}(R)$ generated by $I$ ) contains a nonzero ideal of $Q_{r}(R)$. If $Q_{r}(R)$ is right selfinjective, then $Q_{r}(R)$ is right FPF.

PROOF. Let $M$ be a faithful cyclic $Q_{r}(R)$-module, $g: Q_{r}(R) \rightarrow M$ a $Q_{r}(R)$ epimorphism, and $h: R \rightarrow Q_{r}(R)$ the inclusion homomorphism. Then $g h(R)=N$ is an $R$-module. Let $A=\underline{r}_{R}(N)$. If $A \neq 0$, there exists a nonzero ideal $I$ of $Q_{r}(R)$ such that $I \subseteq A Q_{r}(R)$. Let $H=\{m \in M \mid m I=0\}$. Now $H$ is a $Q_{r}(R)$-submodule of $M$. Hence $H=M$ since $g(1) \in N \subseteq H$. Contradiction! Thus $N$ is a generator in mod- $R$. Hence there exists an $R$-epimorphism $f: \bigoplus_{i=1}^{k} N_{i} \rightarrow R$ where $N_{i}$ is $R$-isomorphic to $N$. From [14, pp. 94-95], there exists a $Q_{r}(R)$-homomorphism $\bar{f}: \bigoplus_{i=1}^{k} M_{i} \rightarrow Q_{r}(R)$ which extends $f$, where $M_{i}$ is $Q_{r}(R)$-isomorphic to $M$. Therefore, there exists $x \in \bigoplus_{i=1}^{k} N_{i}$ such that $f(x)=\bar{f}(x)=1$. Hence $\bar{f}\left(\bigoplus_{i=1}^{k} M_{i}\right)=Q_{r}(R)$. Consequently, $Q_{r}(R)$ is right GFC. By Theorem 2, $Q_{r}(R)$ is right FPF.

COROLLARY 4. Let $R$ be a right nonsingular right GFC ring. Then the following statements are equivalent.

(i) $Q_{r}(R)$ is right $G F C$.

(ii) $Q_{r}(R)$ is FPF.

(iii) If $I$ is a nonzero ideal of $R$, then $I Q_{r}(R)$ contains a nonzero ideal of $Q_{r}(R)$.

(iv) If $I$ is a nonzero ideal of $R$, then $I Q_{r}(R)$ is an essential extension of an ideal of $Q_{r}(R)$.

PROOF. (i) $\Rightarrow$ (ii) follows from Theorem 2 and [8, Corollary 3.14B].

(ii) $\Rightarrow$ (iii). Suppose $I Q_{r}(R)$ contains no nonzero ideals of $Q_{r}(R)$. Let $0 \neq$ $x \in \underline{l}_{Q}\left(I Q_{r}(R)\right) \cap\left(I Q_{r}(R)\right)$ [3, Proposition 1.4]. Since $I$ is essential in $I Q_{r}(R)$ as $R$-modules, there exists $s \in R$ such that $0 \neq x s \in I$. Then $x s \in \underline{l}_{R}(I) \cap I$. But $R$ is semiprime [3, Proposition 1.4]. Contradiction!

(iii) $\Rightarrow$ (i). This is a consequence of Theorem 3 .

(iii) $\Leftrightarrow$ (iv). Suppose condition (iii) holds. Let $X$ be the maximal ideal of $Q_{r}(R)$ contained in $I Q_{r}(R)$. Assume $X$ is not essential in $I Q_{r}(R)$. Let $Y$ be a complement of $X$ in $I Q_{r}(R)$. By [3, Proposition 2.6] there exists a central idempotent $b \in Q_{r}(R)$ such that $Y \subseteq b Q_{r}(R)$ and $X \cap b Q_{r}(R)=0$. Let $B=b Q_{r}(R) \cap I$. Then $B$ is a nonzero ideal in $R$. Hence $B Q_{r}(R)$ contains a nonzero ideal of $Q_{r}(R)$. But this contradicts the maximality of $X$. Therefore, $X$ is essential in $I Q_{r}(R)$. The converse is obvious.

We note that if $R$ is a right nonsingular ring (not necessarily right GFC) which satisfies condition (iii) of Corollary 4 for which $Q_{r}(R)$ is FPF, then $R$ satisfies condition (iv). For example, if $R$ is a right Ore domain which is not right GFC (e.g., a simple Ore domain which is not a division ring $[6 ; 3$, Corollary 1.3$]$ ), then $R$ is a nonsingular ring which satisfies condition (iii) of Corollary 4 for which $Q_{r}(R)$ is 
FPF. The ring $R$ of $2 \times 2$ upper triangular matrices over a field provides an example of a right nonsingular ring which does not satisfy condition (iii) of Corollary 4 even though $Q_{r}(R)$ is FPF. Finally from [3, Proposition 1.4 and Corollary 3.6], we see that a right nonsingular right GFC quasi-Baer (e.g., a right nonsingular right FPF) ring satisfies condition (iii) of Corollary 4. However, there are right nonsingular right GFC rings which satisfy condition (iii) of Corollary 4 but are not quasi-Baer. For example, if $R$ is strongly regular, then $R$ is nonsingular GFC and $R$ satisfies condition (iii) of Corollary 4 since $Q_{r}(R)$ is strongly regular $[\mathbf{1 7}$, p. 254]. But there are strongly regular rings which are not quasi-Baer (e.g., any noncomplete Boolean ring [17, pp. 79 and 253]). Hence Corollary 4 generalizes [3, Theorem 2.8 and Corollary $3.6 ; 8$, Theorem 3.5].

COROLLARY 5. If $R$ is a right GFC ring which satisfies any of the following conditions, then $Q_{r}(R)$ is FPF.

(i) $R$ is right nonsingular and every nonzero ideal of $R$ contains a nonzero central element of $R$.

(ii) Every nonzero ideal of $R$ contains a nonzero central idempotent (e.g., biregular rings).

(iii) $R$ is a P.I. ring which is either semiprime or right nonsingular.

ProOF. Corollary 4 and $[10$, p. 48, Exercise 14] show that the result is true for condition (i). Clearly condition (ii) is a special case of condition (i). From [3, Proposition $1.4 ; 9$, Corollary 2 ; and 16, Theorem 2] it follows that condition (iii) is a special case of condition (i).

We note that in $[\mathbf{1 3}] \mathrm{S}$. Kobayashi has characterized the regular right GFC rings satisfying condition (ii) of Corollary 5 .

\section{REFERENCES}

1. F. W. Anderson and K. R. Fuller, Rings and categories of modules, Springer-Verlag, New York, 1974.

2. G. F. Birkenmeier, Baer rings and quasi-continuous rings have a MDSN, Pacific J. Math. 97 (1981), 283-292.

3. - A generalization of FPF rings (preprint).

4.

5. W. E. Clark, Twisted matrix units semigroup algebras, Duke Math. J. 34 (1967), 417-423.

6. J. H. Cozzens, Homological properties of the ring of differential polymomials, Bull. Amer. Math. Soc. 76 (1970), 75-79.

7. C. Faith, Injective quotient rings of commutative rings, Module Theory, Lecture Notes in Math., vol. 700, Springer-Verlag, New York, 1979, pp. 151-203.

8. C. Faith and S. Page, FPF ring theory: faithful modules and generators of Mod-R, London Math. Soc. Lecture Note Series, no. 88, Cambridge Univ. Press, Cambridge, 1984.

9. J. W. Fisher, Structure of semiprime P. I. rings, Proc. Amer. Math. Soc. 39 (1973), 465-467.

10. K. R. Goodearl, Ring theory: nonsingular rings and modules, Dekker, New York, 1976.

11. , Von Neumann regular rings, Pitman, London, 1979.

12. I. Kaplansky, Rings of operators, Benjamin, New York, 1968.

13. S. Kobayashi, On regular rings whose cyclic faithful modules are generator (preprint).

14. J. Lambek, Lectures on rings and modules, Blaisdell, Waltham, Mass., 1966.

15. A. Pollinger and A. Zaks, On Baer and quasi-Baer rings, Duke Math. J. 37 (1970), 127-138.

16. L. Rowen, Some results on the center of a ring with polymomial identity, Bull. Amer. Math. Soc. 79 (1973), 219-223.

17. B. Stenstrom, Rings of quotients, Springer-Verlag, New York, 1975.

DEPARTMENT OF MATHEMATICS, UNIVERSITY OF SOUTHWESTERN LOUISIANA, LAFAYETTE, LOUISIANA 70504 